rh 19 Revue d'histoire du XIXe siècle

Société d'histoire de la révolution de 1848 et des

révolutions du XIXe siècle

$62 \mid 2021$

Ancêtres

\title{
Corine MAITTE, Didier TERRIER, Les Rythmes du labeur. Enquête sur le temps de travail en Europe occidentale, XIV ${ }^{e}-X I X^{e}$ siècle
}

\section{François Jarrige}

\section{OpenEdition}

\section{Journals}

Édition électronique

URL : https://journals.openedition.org/rh19/7634

DOl : $10.4000 /$ rh 19.7634

ISSN : $1777-5329$

Éditeur

La Société de 1848

\section{Édition imprimée}

Date de publication : 20 juin 2021

Pagination : 260-262

ISSN : 1265-1354

Référence électronique

François Jarrige, «Corine MAITTE, Didier TERRIER, Les Rythmes du labeur. Enquête sur le temps de travail en Europe occidentale, xIve-xIx siècle », Revue d'histoire du XIXe siècle [En ligne], 62 | 2021, mis en ligne le 17 août 2021, consulté le 26 août 2021. URL : http://journals.openedition.org/rh19/7634 ; DOI : https://doi.org/10.4000/rh19.7634

Ce document a été généré automatiquement le 26 août 2021.

Tous droits réservés 


\title{
Corine MAITTE, Didier TERRIER, Les Rythmes du labeur. Enquête sur le temps de travail en Europe occidentale, XIV ${ }^{e}$ XIX $X^{e}$ siècle
}

\author{
François Jarrige
}

\section{RÉFÉRENCE}

Corine MAITTE, Didier TERRIER, Les Rythmes du labeur. Enquête sur le temps de travail en Europe occidentale, XIV ${ }^{e}-X I X^{e}$ siècle, Paris, La Dispute, coll. « Travail et salariat », 2020, 432 p., $28 €$.

1 La publication de l'enquête de Corine Maitte et Didier Terrier sur les «rythmes du labeur » et la question du temps de travail en Europe était attendue. Fruit d'un travail de longue haleine, l'ouvrage offre une synthèse importante, qui conclut plusieurs années de recherches individuelles et collectives, et revient sur des questions aussi anciennes que controversées de l'historiographie ${ }^{1}$. L'une des grandes forces et originalités de l'étude réside dans le choix de la longue durée englobant dans un même mouvement les transformations qui s'étalent de la fin du Moyen Âge à la fin du $\mathrm{XIX}^{\mathrm{e}}$ siècle. Elle réside aussi dans le choix de sortir des approches statistiques trop macros, des fausses évidences quantitatives pour suivre les activités concrètes et les expériences vécues des temporalités du travail, en accordant toute leur place à la diversité des contextes et des situations individuelles. Pour cela, les auteurs ont mobilisé une bibliographie et des enquêtes archivistiques impressionnantes, qui témoignent de leur connaissance intime des réalités des mondes du travail anciens.

2 Les auteurs remettent ainsi en cause nombre d'idées reçues, comme celle imaginant un âge d'or préindustriel où les travailleurs œuvraient à leur rythme. Ils montrent aussi que le travail, qu'il soit rémunéré ou non, n'a cessé d'être traversé par la question du 
temps. Depuis Karl Marx et Max Weber, la rationalité temporelle a en effet été considérée comme une caractéristique fondamentale du capitalisme moderne. Lewis Mumford faisait ainsi de l'horloge l'élément capital de l'ère industrielle, alors que le Britannique Edward Palmer Thompson distinguait de son côté l'ancien travail mesuré à la tâche et celui de l'âge industriel qui serait de plus en plus mesuré par le temps. Dans le travail industriel moderne le temps devient l'élément fondamental du système d'exploitation. Maitte et Terrier se placent dans les pas de ces réflexions, tout en contestant les approches trop englobantes ou simplificatrices, trop éloignées des terrains concrets. Sans être réellement comparatiste, leur enquête se déploie à l'échelle européenne, explorant des situations de travail diverses, dans la péninsule italienne pour l'époque moderne, mais surtout en Angleterre, en France et en Belgique, pays qui sont au cœur de l'industrialisation du $\mathrm{XIX}^{\mathrm{e}}$ siècle. Elle se déploie en trois parties thématiques qui interrogent d'abord ce qu'est une journée de travail, puis les temps du travail « de la semaine à l'année ", avant d'explorer le contenu du travail concret, entre rationalisation/intensification des tâches et autonomie de la main-d'œuvre. L'étude est si riche et foisonnante qu'elle se laisse difficilement résumer en quelques lignes. La question des temps du travail est en effet rendue complexe du fait de la multitude des situations selon les groupes professionnels ou les régions, et de la nécessité d'explorer des enjeux aussi divers que le rôle de l'État, les rémunérations, à la tâche ou à façon, les formes d'encadrement, les fêtes religieuses, l'impact du machinisme et de l'usine, etc.

3 Longtemps l'histoire du temps de travail a été ramenée à celle de sa diminution progressive depuis le $\mathrm{XIX}^{\mathrm{e}}$ siècle. Pourtant, les temps du travail renvoient à des rythmes et des enjeux bien plus divers, difficilement réductibles à un modèle unique, selon qu'on s'inscrit dans un cadre proto-industriel, sur un chantier, dans les petits ateliers urbains ou les nouvelles usines mécanisées. La question se pose à plusieurs échelles et implique de prendre en compte la diversité des groupes et les types de durée, en distinguant notamment les rythmes journaliers, hebdomadaires, saisonniers, ou annuels, très différents entre eux. Interroger les temps du travail implique aussi de penser à la fois les rythmes individuels des gestes et de l'effort qui peuvent varier d'un moment à l'autre, d'une activité à l'autre, et les cadres normatifs imposés par les fabricants et l'État afin d'homogénéiser des situations variables. Il faut en effet rappeler que longtemps en Europe on ne compte pas le temps et on travaille tant qu'il le faut. L'uniformisation horaire est tardive et avant le $\mathrm{XIX}^{\mathrm{e}}$ siècle c'est souvent la position du soleil qui détermine les heures.

4 Si la question des rythmes du travail est déjà ancienne et se pose avant l'industrialisation du $\mathrm{XIX}^{\mathrm{e}}$ siècle, elle ne cesse de prendre de l'ampleur et de devenir un enjeu récurrent dès lors que le travail devient de plus en plus une marchandise mesurée et calculée de plus en plus précisément. De ce point de vue, le XIX siècle ne constitue pas une rupture aussi nette qu'on l'a dit. Il ne voit sans doute pas d'allongement massif des journées, déjà très longues depuis longtemps. La discipline qui tente d'imposer un temps de travail effectif continu est ancienne. Replacer l'évolution du temps annuel de travail dans la longue durée permet ainsi de déconstruire l'idée communément admise d'une évolution linéaire généralement résumée en trois phases : une longue phase préindustrielle (avant le XIX ${ }^{\mathrm{e}}$ siècle) durant laquelle on ne travaillerait guère plus de 1500 à 2000 heures, une brève et forte augmentation des durées au début de l'industrialisation, culminant autour de 
3000 heures autour de 1830, puis une lente décrue à partir des années 1830-1840, pour arriver aux évaluations actuelles (autour de 1600 heures).

Ce type d'analyse linéaire, dans laquelle le $\mathrm{XIX}^{\mathrm{e}}$ siècle marque un moment de basculement et de transformation majeur, marqué par l'allongement des durées dans un premier temps, avant leur réduction sous la pression des organisations ouvrières et de l'intervention croissante des États, tend à simplifier à l'excès des situations plus complexes selon les auteurs. Ainsi un ouvrier du XVIII ${ }^{\mathrm{e}}$ siècle pouvait travailler plus de 4000 heures, et une partie importante de ceux de la fin du XIX ${ }^{\mathrm{e}}$ siècle continue souvent à trimer plus de 3000 heures par an. Il faut attendre la période 1890-1920 pour constater l'amorce d'un changement réel, notamment avec l'adoption des grandes lois sur la journée de huit heures. La diversité des situations en fonction des secteurs, des périodes, des types de travaux, et l'ampleur des possibles font par ailleurs de la durée du travail un enjeu très individualisé, procédant parfois d'un choix, souvent d'une contrainte qu'il est impossible de ramener à quelques moyennes ou à des évolutions linéaires. Dans ce domaine plus qu'en aucun autre, constatent les auteurs, «les moyennes sont de vrais outrages infligés aux individus réels » (p. 391). Les historiens rejoignent dès lors le constat de nombreux sociologues du travail qui insistent sur la nécessité d'explorer le contenu du temps du travail et la diversité des rythmes de l'activité productive, au-delà des normes proclamées et des moyennes factices.

6 Les auteurs font aussi des rythmes du travail et de ses durées des enjeux de luttes et de négociations incessants. Ils citent par exemple cette pétition que des ouvriers de Tourcoing adressent en 1884 au préfet, pour dénoncer avec véhémence les «abus que font les filateurs » qui les contraignent à travailler 13 voire 14 heures dans des ateliers surchauffés, et qui «augmentent toujours les vitesses de leur machine et de leur métier»(p.361). Ces travailleurs du textile dénoncent les cadences infernales et demandent que la durée du travail soit limitée à 12 heures, ce qui est " déjà beaucoup » ajoutent-ils. À cette date pourtant, la durée du travail était déjà théoriquement limitée à 12 heures en France dans les usines, mais comme dans les autres pays européens, les législations encadrant les temps du travail peinent à être appliquées réellement. Ce témoignage montre toute l'importance de la question des temporalités et des rythmes du travail pour la main-d'œuvre soumise à l'accroissement des cadences.

7 Pour les auteurs, les rythmes du travail sont des observatoires privilégiés des mutations du capitalisme. Discipliner les travailleurs, c'est en effet imposer un nombre d'heures de travail, mais aussi s'assurer qu'aucun temps laborieux n'est gaspillé. C'est pourquoi la diminution de la durée peut aller de pair avec une densification qui engendre un surcroît de fatigue et une usure précoce, maux désormais bien connus et qui expliquent l'essor actuel des souffrances au travail et les cas de "burn out», phénomènes qu'on observe déjà dans les usines mécanisées de la fin du XIX ${ }^{\mathrm{e}}$ siècle. En rouvrant la diversité des enjeux soulevés par la question des rythmes du travail, les auteurs interrogent en définitive les rapports de domination dans les sociétés capitalistes qui imposent leurs rythmes infernaux. Ils concluent d'ailleurs leur belle enquête par une réflexion sur ce que pourraient être les rythmes de vie et de travail dans une société de « décroissance soutenable » où le travail serait mis au service du bien commun au lieu d'être pensé comme un facteur d'enrichissement de quelques-uns. 


\section{NOTES}

1. Notamment dans un dossier de revue qu'ils ont coordonné dès 2011 : Corine Maitte, Didier Terrier (dir.), «Temps de travail », Genèses, n 85, 2011/4; et un ouvrage collectif paru en 2014 : Corinne Maitte, Didier Terrier (dir.), Les Temps du travail. Normes, pratiques, évolutions (XIVeXIX ${ }^{e}$ siècle), Rennes, Presses universitaires de Rennes, coll. « Pour une histoire du travail », 2014. 